\title{
Lamotrigine and lithium are effective maintenance treatments in recently depressed people with bipolar I disorder
}

Calabrese JR, Bowden CL, Sachs G, et al. A placebo-controlled 18-month trial of lamotrigine and lithium maintenance treatment in recently depressed patients with bipolar I disorder. J Clin Psychiatry 2003;64:1013-24.

What are the effects of lamotrigine and lithium for maintenance treatment in recently depressed people with bipolar I disorder?

\section{METHODS}

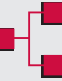

Design: Randomised controlled trial.

Q⿻

Allocation: Unclear.

Blinding: Double blind.

Follow up period: 18 months.

Setting: 79 centres in 15 countries; 1997 to 2001.

Patients: 463 adults with bipolar I disorder (mean age range 42 to 44 years), who were stabilised with lamotrigine during an 816 week open label phase $(n=966)$. Main inclusion criteria: current or recent (past 60 days) DSM-IV major depressive episode; $\geqslant 1$ manic or hypomanic episodes, and $\geqslant 1$ depressive episodes within 3 years of enrolment. Exclusions: >6 DSM-IV mood episodes in the past year; significant psychiatric or medical comorbidity; epilepsy or suicidal. All other psychotropic medicines were discontinued before randomisation.

$\mathbf{R}_{\mathbf{2}}$

Intervention: Maintenance treatment with lamotrigine 150,200 or $400 \mathrm{mg} /$ day), lithium (serum levels $0.8-1.1 \mathrm{mEq} / \mathrm{l}$ ) or placebo.

国典

Outcomes: Time to intervention for any mood episode; adverse events.

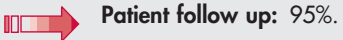

\section{MAIN RESULTS}

Both lamotrigine (200 or $400 \mathrm{mg} /$ day) and lithium significantly increased the time to intervention for any mood episode compared with placebo (200 days with lamotrigine $v 170$ days with lithium $v$ 93 days with placebo; $\mathrm{p}=0.029$ for both comparisons $v$ placebo). There was no significant difference in the time to intervention between lamotrigine and lithium $(p=0.915) .11 \%$ of participants withdrew due to adverse effects (see table for most common adverse events).

\section{CONCLUSIONS}

Lamotrigine and lithium are better than placebo for preventing mood episodes in people with bipolar I disorder.

For correspondence: J Calabrese, University Hospitals of Cleveland/Case Western Reserve School of Medicine, Department of Psychiatry, Cleveland, Ohio, USA; jcr8@po.cwru.edu

Sources of funding: GlaxoSmithKline, Abbott Laboratories, Bristol-Myers Squibb, GlaxoWellcome, Janssen, Lilly Research, the National Institute of Mental Health, Parke-Davis, R.W. Johnson Pharmaceutical Institute, Smith Kline Beecham, the Stanley Foundation, Eli Lilly, Solvay, Novartis, Sanofi, Astra Zeneca, and Pfizer.

\section{NOTE}

There were initially three lamotrigine treatment groups; enrolment was stopped in the $50 \mathrm{mg} / \mathrm{day}$ and $400 \mathrm{mg} /$ day groups during the study because of poor enrolment. Efficacy data for lamotrigine was pooled for the $200 \mathrm{mg}$ and $400 \mathrm{mg} /$ day groups, a decision that had been made a priori. Authors point out that participants who were intolerant or unresponsive to lamotrigine would have been excluded during the prerandomisation stabilisation period, and this may have biased results.

\section{Commentary}

This study investigates treatment with lamotrigine after an acute response to the drug in recently depressed subjects with bipolar disorder. This issue is clinically important, given the depressive burden of bipolar disorder. The design is that of a randomised double blind placebo controlled clinical trial comparing five groups: three doses regimens of lamotrigine $(50,200,400 \mathrm{mg} /$ day), lithium and placebo.

Randomisation took place after 463 patients were stabilised on lamotrigine during an open label phase of treatment for acute major depression. Data analyses show lamotrigine and lithium being superior to placebo in preventing additional treatment interventions (the primary outcome) because of the occurrence of a mood episode.

This study is not a classic prophylaxis study: in that design, patients who have become euthymic in any manner (recently depressed, recently manic, or euthymic for a long time due to natural history) enter the study. This design is rather one of relapse prevention (or an "enriched" design), in which all patients who were randomised to maintenance treatment initially responded to lamotrigine (with or without other psychotropics) for an acute major depressive episode. In other words, the generalisability of these findings is limited to such patients, and not all patients who reach the maintenance phase in other ways.

Also, lithium is included as an active control-that is, as a test of assay sensitivity. As the study is not designed and powered to assess lithium efficacy, definitive conclusions about lithium's efficacy cannot be made. Thus, it would not exactly follow from these data that lithium is not effective in prevention of depressive episodes (one of the secondary analyses). Rather, the primary conclusion one could draw is that lamotrigine is effective in delaying relapse of mood episodes, particularly of the depressive subtype, with the above constraints of generalisability. Federico Soldani, MD MSc, S Nassir Ghaemi, MD MPH Bipolar Disorder Research Program, Department of Psychiatry, Cambridge Health Alliance, Harvard Medical School, Cambridge, Massachusetts, USA

Table Common adverse events occurring during treatment, $\mathrm{n}(\%)$

\begin{tabular}{lccc}
\hline $\begin{array}{l}\text { Adverse } \\
\text { event }\end{array}$ & $\begin{array}{l}\text { Lamotrigine } \\
(\mathbf{n}=169)\end{array}$ & $\begin{array}{l}\text { Lithium } \\
(\mathbf{n}=120)\end{array}$ & $\begin{array}{l}\text { Placebo } \\
(\mathbf{n}=121)\end{array}$ \\
\hline Headache & $30(18)$ & $23(19)$ & $25(21)$ \\
Nausea & $28(17)$ & $24(20)$ & $15(12)$ \\
Tremor & $9(5)^{*}$ & $20(17) \dagger$ & $6(5)$ \\
Rash & $12(7) \dagger$ & $5(4)$ & $3(2)$ \\
Dizziness & $21(12)$ & $13(11)$ & $12(10)$ \\
\hline
\end{tabular}

${ }^{*} \mathrm{p}<0.05$ versus lithium

$\mathrm{tp}<0.05$ versus placebo. 